\title{
The presence of risk factors and corresponding treatment strategies post-surgical resection in stage IV hypopharyngeal squamous cell carcinoma patients: a retrospective cohort study
}

\author{
Yu Heng ${ }^{1 \#}$, Xiaoke Zhu ${ }^{1 \#}$, Liang Zhou ${ }^{1}$, Ming Zhang $^{1}$, Hong Zhou $^{2}$, Lei Tao ${ }^{1}$ \\ ${ }^{1}$ Department of Otolaryngology, Shanghai Key Clinical Disciplines of Otorhinolaryngology, Eye Ear Nose \& Throat Hospital, Fudan University, \\ Shanghai 200031, China; ${ }^{2}$ Department of Otolaryngology, Fudan University Pudong Medical Center, Shanghai 200031, China \\ Contributions: (I) Conception and design: Y Heng, H Zhou, L Tao; (II) Administrative support: None; (III) Provision of study materials or patients: \\ None; (IV) Collection and assembly of data: All authors; (V) Data analysis and interpretation: All authors; (VI) Manuscript writing: All authors; (VII) \\ Final approval of manuscript: All authors. \\ \#These authors contributed equally to this work. \\ Correspondence to: Lei Tao, PhD. Department of Otolaryngology, Eye Ear Nose \& Throat Hospital, Fudan University, 83 Fenyang Road, Shanghai \\ 200031, China. Email: doctortaolei@163.com; Hong Zhou, PhD. Department of Otolaryngology, Fudan University Pudong Medical Center, \\ Shanghai 200090, China. Email: zhsxai@163.com.
}

Background: This study aims to explore the adverse features and determine whether adjuvant chemoradiation after surgical resection can benefit stage IV hypopharyngeal squamous cell carcinoma (HSCC) patients.

Methods: We conducted a retrospective review covering 267 patients with stage IV HSCC. Propensity score-matched analysis was employed to reduce selection bias.

Results: T3-T4 or N2c-N3 stage, positive surgical margin, extracapsular spread and lymphovascular invasion were adverse features for overall survival (OS) in stage IV HSCC patients. For patients possessing these adverse features, those who received postoperative adjuvant treatment (PAT) had significantly better OS and recurrence-free survival (RFS) than patients who did not ( $\mathrm{P}$ value $=0.000$ and 0.007 , respectively). In addition, adjuvant chemoradiation demonstrated better OS and RFS compared to adjuvant radiation ( $\mathrm{P}$ value $=0.030$ and 0.017 , respectively). However, PAT showed no significant impact on OS and RFS (P value $=0.776$ and 0.847 , respectively) in patients without adverse features.

Conclusions: Adjuvant treatments are recommended for stage IV HSCC patients that possess adverse features of pT3 and pT4 stages, N2c and N3 stages, positive surgical margin, extracapsular spread and lymphovascular invasion. For these patients, postoperative adjuvant chemoradiotherapy (CRT) is preferred. For patients without adverse features, observation and regular re-examination is sufficient post tumour resection.

Keywords: hypopharyngeal squamous cell carcinoma (HSCC); stage IV; adverse features; postoperative adjuvant treatment (PAT); survival outcome

Submitted Oct 13, 2019. Accepted for publication Jan 03, 2020.

doi: 10.21037/atm.2020.01.102

View this article at: http://dx.doi.org/10.21037/atm.2020.01.102

\section{Introduction}

Hypopharyngeal squamous cell carcinoma (HSCC) is a rare entity accounting for less than $5 \%$ of all head and neck squamous cell carcinomas (HNSCC) (1). However,
HSCC has a poorer prognosis than other types of HNSCC due to being asymptomatic during earlier stages and are caught only during advanced stages for most patients. The rich lymphatic network in the pharyngeal region and the 
propensity for submucosal spread leads to higher risk in lymph node metastases, with reported statistics of $60-80 \%$ ipsilateral nodal metastasis, and $40 \%$ contralateral occult nodal metastasis (2-4). Over $50 \%$ of patients present with stage IV HSCC, and 5-year survival for stage III/IV HSCC hovers at $15-45 \%(1,5)$. As HSCC is relatively rare amongst all head and neck cancers, there is a lack of studies with significant stage IV HSCC sample size, and optimal treatment strategies for patients with stage IV HSCC remain controversial.

In recent years, definitive chemoradiotherapy (CRT) and induction chemotherapy followed by radiotherapy (RT) have been introduced as new strategies preferable over surgery for many types of advanced HNSCC (6-8), including advanced HSCC (5,9-12). These non-surgical treatments offer patients the opportunity to preserve their laryngeal function. Considering the extremely poor prognosis for all stages of HSCC, further trials are required to assess the oncological outcome of non-surgical strategies. However, few previous studies have focused on stage IV HSCC, especially those with adverse features. Patients with stage IV HSCC have a significant tendency to relapse, yet the tissue damage due to RT makes salvage surgery rather difficult, leading to higher morbidity when compared to patients with other types of HNSCC (13). Thus, surgery-based treatment remains the most common therapeutic strategy in China. The surgery includes laryngopharyngectomy plus neck dissection, with optional PATs. Although the National Comprehensive Cancer Network (NCCN) guidelines recommend both adjuvant RT and CRT for stage IV HSCC patients following tumour resection (14), it remains unknown if adjuvant chemotherapy can benefit patients with high-risk stage IV HSCC. To investigate this, our study compares the clinical outcomes of surgery alone and surgery followed by RT or CRT in patients with stage IV HSCC.

\section{Methods}

The study has been submitted to Chinese Clinical Trial Registry chictr.org.cn. The assigned Unique Identifying Number is ChiCTR1900022847.

\section{Study population}

Between 2003 and 2015, 553 patients with untreated HSCC received both primary tumour resection and neck dissection at Department of Otorhinolaryngology, Head and Neck Surgery at the Eye, Ear, Nose and Throat Hospital of Fudan University. Among them, 332 (60.0\%) patients had stage IV HSCC as confirmed by postoperative pathology. Patients were excluded if they had multiple primary hypopharyngeal carcinoma or distant metastasis when diagnosed, has received any neoadjuvant RT or chemotherapy, or were lost to follow-up. Consequently, 267 patients were included in our statistical analysis (Figure 1). This study was approved by the Institutional Ethics Committee of the Eye \& ENT Hospital of Fudan University. All participants gave informed consent to take part in the study.

\section{Collected information}

We reviewed the patients' medical records to acquire their baseline clinical and disease characteristics, including sex, age, smoking history, use of alcohol, tumour location, surgical options and location of neck dissection, grade of differentiation, depth of tumour invasion, number of lymphatic metastases, lymphovascular invasion, extracapsular spread, fixation of hemilarynx, and adjuvant treatment. A multidisciplinary team re-evaluated the patients every 3 to 4 months for the first year after completion of treatment, and every 6 months thereafter. Each follow-up included a physical examination, blood test, laryngeal and gastrointestinal endoscopy, chest radiography, and computed tomography (CT) or magnetic resonance imaging (MRI) of the neck and chest. Overall survival (OS) and recurrence-free survival (RFS) were used as the end events of this study; data on site of recurrence, time to recurrence and death were also obtained during the follow-up.

\section{Treatment methods}

All patients received surgical treatment, with recommendation for postoperative adjuvant therapies made clear. However, taking patient's postoperative status such as low KPS score and patient's preference into consideration, some received surgical treatment only. Three classifications were thus established: surgery alone, surgery plus postoperative radiotherapy (pRT) and surgery plus postoperative chemoradiotherapy (pCRT).

Postoperative adjuvant therapy included $\mathrm{pRT}$ and pCRT. pRT was conducted with a fractionation of 2 Gy/ fraction once a day, five times a week. The curative dose was 66-70 Gy for both the primary tumour site and the involved 


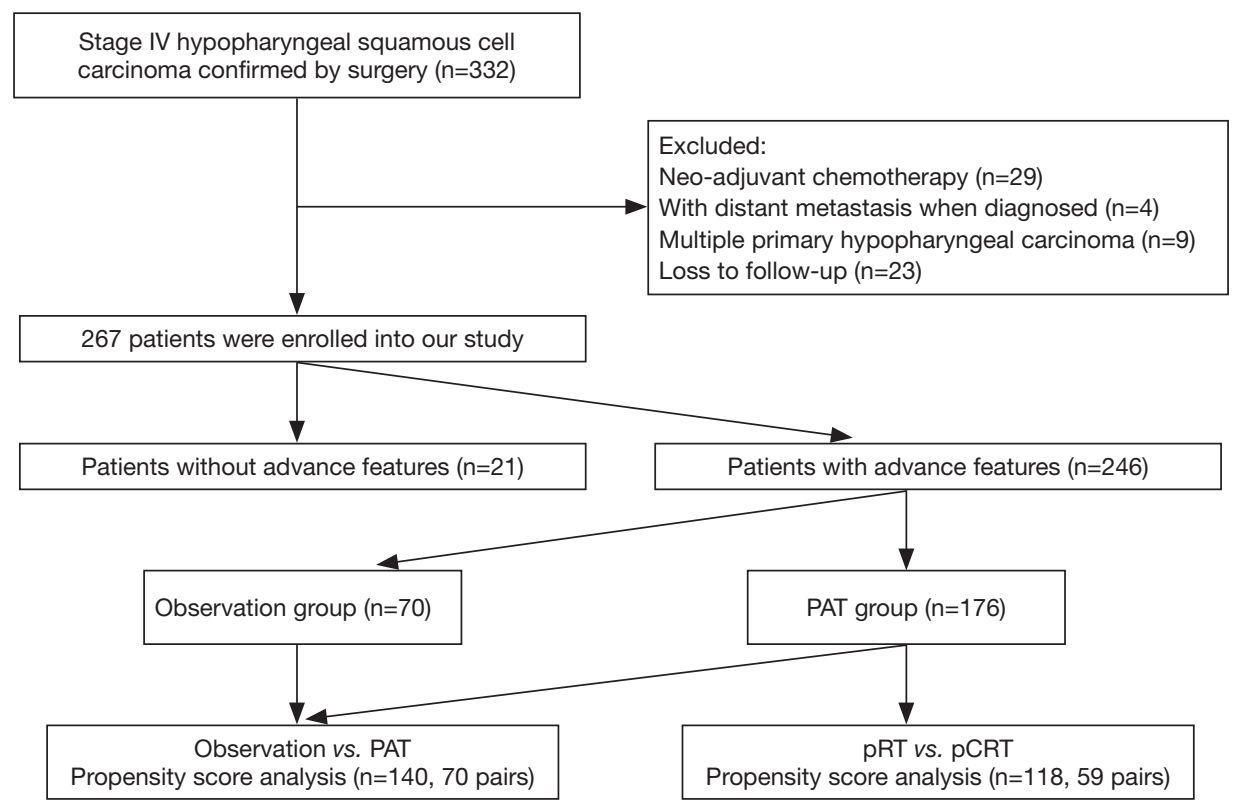

Figure 1 Flow diagram of case selection steps. PAT, postoperative adjuvant treatment; pRT, postoperative radiotherapy; pCRT, postoperative chemoradiotherapy.

lymph nodes (including possible local subclinical infiltration of the primary site and high-risk lymph nodes).

The preferred pCRT strategy for local advanced HNSCC is concurrent chemoradiotherapy (CCRT), which is platinum-based. Most patients received a DDP regimen where cisplatin (DDP) $(45-50 \mathrm{mg} /$ day) was delivered across 3 consecutive days on day $1-3$, day 22-24, and day 43-45, with an average total amount of $197.9 \mathrm{mg} / \mathrm{m} 2$ cisplatin administered to patients receiving this regimen in our study. Two other patients received PF regimen consisting of cisplatin and 5-Fluorouracil (5-FU) in our study, and were also enrolled in our research.

While most patients received CCRT, another pCRT approach, sequential chemoradiotherapy (seq-CRT), was used at our institution for a few patients. In seq-CRT, platinum-based induction chemotherapy was applied, followed by radiation-based locoregional strategy.

\section{Statistical analysis}

OS was defined as the time from the first date of treatment to the last contact or death of any cause. RFS was defined as the time from the first date of treatment to local/regional recurrence or distant metastasis. Univariate analysis was used to evaluate the tumour characteristics to obtain the prognostic values of $\mathrm{OS}$; variables with $\mathrm{P}$ values $<0.10$ in the univariate analysis were investigated using multivariate Cox regression to determine the impact of adverse features on survival outcomes. Propensity score matching (PSM) was used to balance the patients' characteristics, including age, pT stage, pN stage, smoking history, drinking history, differentiation degree, lymph nodal fusion, cervical nodal necrosis, fixation of hemilarynx, and internal jugular vein adhesion with the aim of collecting bias of the pRT group and the pCRT group. We adopted a caliper width of 0.2 of standard deviation for the nearest neighbour matching; the 1:1 matching method was conducted using the MatchIt package of R (version 3.5.1; R Development Core Team).

Survival rates (including OS and RFS) were calculated at 5 years. Actuarial OS and RFS rates were calculated according to the Kaplan-Meier method using SPSS 24.0 software (SPSS Inc., Chicago, IL, USA). Hazard ratio and the relative $95 \%$ confidence interval (CI) were also calculated. Statistical differences between the different treatment strategies were calculated using the log-rank test, and a $\mathrm{P}$ value $<0.05$ was considered statistically significant.

\section{Results}

\section{Patients and characteristics}

A total of 267 patients were included in this study. The 
patients and characteristics are presented in Table 1. The median OS of all the patients in this study was 33.5 months (range, 2.0-148.0 months). All patients underwent surgical treatment, including both primary tumour resection and neck dissection. Patients' treatment characteristics are shown in Table S1.

\section{Treatment outcomes}

Five-year OS and RFS for all patients were $37.5 \%$ and $35.3 \%$, respectively. Kaplan-Meier analysis results for OS in patients with stages $\mathrm{T} 1, \mathrm{~T} 2, \mathrm{~T} 3$ and $\mathrm{T} 4$ are shown in Figure $S 1 A, B$. OS was lower for patients with stages T3/ $\mathrm{T} 4$ than for those with stages T1/T2 (P value $<0.0001$ ); however, no difference in OS was observed between stages $\mathrm{T} 1$ and $\mathrm{T} 2$, and stages $\mathrm{T} 3$ and $\mathrm{T} 4$ ( $\mathrm{P}$ value $=0.896$ and 0.204 , respectively). OS for stages N0, N1, N2 and N3 are shown in Figure S1C,D. OS are higher for stages N0$\mathrm{N} 2 \mathrm{~b}$ than stages $\mathrm{N} 2 \mathrm{c} / \mathrm{N} 3$ for all patients $(\mathrm{P}$ value $=0.0076$, Figure S1E); this was also observed for patients with stages $\mathrm{T} 1$ and $\mathrm{T} 2$, and stages $\mathrm{T} 3$ and $\mathrm{T} 4(\mathrm{P}$ value $=0.0209$ and 0.0085 , respectively. Figure $S 1 F, G, H, I)$.

\section{Adverse features of patients with stage IV HSCC}

We conducted a univariate analysis to determine the variables that influence OS. Age (HR, 0.742; 95\% CI, $0.517-1.066$; $\mathrm{P}$ value $=0.102$ ), history of smoking (HR, $0.855 ; 95 \%$ CI, 0.588-1.243; P value $=0.412$ ), history of drinking alcohol (HR, 0.991; 95\% CI, 0.687-1.431; P value $=0.963)$, tumor differentiation $(\mathrm{HR}, 1.002 ; 95 \% \mathrm{CI}$, $0.592-1.697 ; \mathrm{P}$ value $=0.993)$, tumor invasive depth $(\mathrm{HR}$, $1.242 ; 95 \%$ CI, 0.804-1.918; $\mathrm{P}$ value $=0.329$ ), cervical nodal necrosis (HR, 1.236; 95\% CI, 0.854-1.790; $\mathrm{P}$ value $=0.261$ ), fixation of hemilarynx (HR, 1.346; 95\% CI, 0.940-1.927; $\mathrm{P}$ value $=0.105)$, and thyroid gland invasion (HR, 1.612; 95\% CI, 0.889-2.923; P value $=0.116$ ) were excluded by univariate analysis. Multivariate analysis was conducted to determine whether the factor showed a $\mathrm{P}$ value $<0.100$ in the univariate analysis, as shown in Figure 2. The results suggest that patients with stages T3-T4 (HR, 3.808; 95\% CI, 2.163-6.704; $\mathrm{P}$ value $=0.000)$ or $\mathrm{N} 2 \mathrm{c}-\mathrm{N} 3$ stages $(\mathrm{HR}$, $1.958 ; 95 \%$ CI, 1.275-3.006; $\mathrm{P}$ value $=0.001)$, positive surgical margin (HR, 1.916; 95\% CI, 1.161-3.162; P value $=0.011)$, extracapsular spread $(\mathrm{HR}, 1.543 ; 95 \% \mathrm{CI}$, $1.042-2.467 ; \mathrm{P}$ value $=0.035)$ and lymphovascular invasion $(\mathrm{HR}, 1.666 ; 95 \% \mathrm{CI}, 1.050-2.644 ; \mathrm{P}$ value $=0.030) \mathrm{had}$ significantly worse survival outcomes. These five variables could be adverse features for patients with stage IV HSCC.

\section{Survival outcomes based on whether a patient received PAT or not}

Based on the results of the analysis presented above, the patients were divided into two groups: those with adverse features and those without adverse features. Thus, 21 patients were assigned to the non-adverse features group and 246 patients were assigned to the adverse features group. In the non-adverse features group, 13 of the 21 patients received PAT, including pRT and pCRT. In the non-adverse features group, no statistically significant difference in OS and RFS was found for the patients receiving adjuvant treatment and those not receiving it $(\mathrm{P}$ value $=0.776$ and 0.847 , respectively), as seen in Figure 3. In the adverse features group, 176 of the 246 patients received PAT and 70 received surgical treatment only. To verify whether PAT could improve the overall survival of stage IV HSCC patients with adverse features, we used PSM analysis to balance the other clinical characteristics, including age, alcohol drinking history, smoking history, tumour differentiation, lymph nodal fusion, tumour invasive depth, cervical nodal necrosis, fixation of hemilarynx, and internal jugular vein adhesion. Consequently, we included 70 pairs of patients in the analysis (Table 2). The multivariate analysis results for the matched patients are shown in Figure 4, indicating that PAT was associated with increased OS (HR, 0.337; 95\% CI, $0.193-0.587$; $\mathrm{P}$ value $=0.000)$ and RFS $(\mathrm{HR}, 0.450 ; 95 \% \mathrm{CI}$, $0.252-0.806$; $\mathrm{P}$ value $=0.007)$ in stage IV HSCC patients with adverse features.

\section{Comparison of two different PAT strategies}

Among patients receiving PAT, an additional PSM analysis was conducted to compare the clinical outcomes of surgery followed by RT and CRT. The basic characteristics of the matched patients are shown in Table 2. The multivariate analysis results for the matched pRT and pCRT patients, presented in Figure 5, showed that the pCRT group had better OS (HR, 0.539; 95\% CI, 0.308-0.942; P value $=0.030)$ and better RFS (HR, 0.518; 95\% CI, 0.301-0.890; $\mathrm{P}$ value $=0.017)$ than the pRT group.

\section{Locoregional and distant control}

The patterns for recurrence in all patients are summarised in Table S2. For patients with tumour recurrence, locoregional recurrence was the most common type of recurrence; it accounted for $53.9 \%$ of the cases, followed by distant metastases (33.8\%). The lung was the most common site of distant metastases for patients with stage IV HSCC. 
Table 1 Patients and characteristics

\begin{tabular}{|c|c|c|}
\hline Characteristics & $\mathrm{n}$ & $\%$ \\
\hline \multicolumn{3}{|l|}{ Age } \\
\hline$<60$ & 147 & 55.1 \\
\hline$\geq 60$ & 120 & 44.9 \\
\hline \multicolumn{3}{|l|}{ History of smoking } \\
\hline Yes & 175 & 65.5 \\
\hline No & 92 & 34.5 \\
\hline \multicolumn{3}{|l|}{ History of drinking alcohol } \\
\hline Yes & 154 & 57.7 \\
\hline No & 113 & 42.3 \\
\hline \multicolumn{3}{|l|}{ Primary site } \\
\hline Pryform sinus & 223 & 83.5 \\
\hline Posterior pharyngeal wall & 30 & 11.2 \\
\hline Postcricoid region & 14 & 5.2 \\
\hline \multicolumn{3}{|l|}{ T-classification } \\
\hline $\mathrm{T} 1$ & 9 & 3.4 \\
\hline $\mathrm{T} 2$ & 56 & 21.0 \\
\hline T3 & 96 & 36.0 \\
\hline $\mathrm{T} 4$ & 106 & 39.7 \\
\hline \multicolumn{3}{|l|}{$\mathrm{N}$-classification } \\
\hline No & 21 & 7.9 \\
\hline N1 & 22 & 8.2 \\
\hline N2 & 183 & 68.5 \\
\hline N3 & 42 & 15.7 \\
\hline \multicolumn{3}{|l|}{ Pathological stage } \\
\hline IVa & 222 & 83.1 \\
\hline $\mathrm{IVb}$ & 45 & 16.9 \\
\hline \multicolumn{3}{|l|}{ Tumor differentiation } \\
\hline Well or moderately & 242 & 90.6 \\
\hline Poorly & 25 & 9.4 \\
\hline \multicolumn{3}{|l|}{ Extracapsular spread } \\
\hline Presence & 112 & 41.9 \\
\hline Absence & 155 & 58.1 \\
\hline \multicolumn{3}{|l|}{ Lymphovascular invasion } \\
\hline Presence & 41 & 15.4 \\
\hline Absence & 226 & 84.6 \\
\hline
\end{tabular}

Table 1 (continued)
Table 1 (continued)

\begin{tabular}{|c|c|c|}
\hline Characteristics & $\mathrm{n}$ & $\%$ \\
\hline \multicolumn{3}{|l|}{ Tumor invasive depth } \\
\hline Muscular layer & 50 & 18.7 \\
\hline No muscular layer & 217 & 81.3 \\
\hline \multicolumn{3}{|c|}{ Cervical nodal necrosis } \\
\hline Yes & 91 & 34.1 \\
\hline No & 176 & 65.9 \\
\hline \multicolumn{3}{|l|}{ Fixation of hemilarynx } \\
\hline Yes & 118 & 44.2 \\
\hline Limited & 45 & 16.9 \\
\hline No & 104 & 39.0 \\
\hline \multicolumn{3}{|l|}{ Thyroid gland invasion } \\
\hline Yes & 33 & 12.4 \\
\hline No & 234 & 87.6 \\
\hline \multicolumn{3}{|l|}{ Esophageal invasion } \\
\hline Yes & 30 & 11.2 \\
\hline No & 237 & 88.8 \\
\hline \multicolumn{3}{|l|}{ Lymph nodal fusion } \\
\hline Yes & 134 & 50.2 \\
\hline No & 133 & 49.8 \\
\hline \multicolumn{3}{|c|}{ Internal jugular vein adhesion } \\
\hline Yes & 115 & 43.1 \\
\hline No & 152 & 56.9 \\
\hline \multicolumn{3}{|c|}{ Positive surgical margin } \\
\hline Presence & 35 & 13.1 \\
\hline Absence & 232 & 86.9 \\
\hline
\end{tabular}

\section{Discussion}

Although survival has improved for patients with HSCC over the past three decades (15), the prognosis of patients with this disease remains unfavourable, especially for those with stage IV disease (16). Given the rarity of tumours in this region, very few studies have investigated large sample of patients in advanced stages; thus, research on stage IV HSCC is urgently needed to establish optimal strategy.

In recent years, the trend of using RT instead of surgical treatment has resulted in high rates of laryngeal preservation along with survival outcomes comparable 


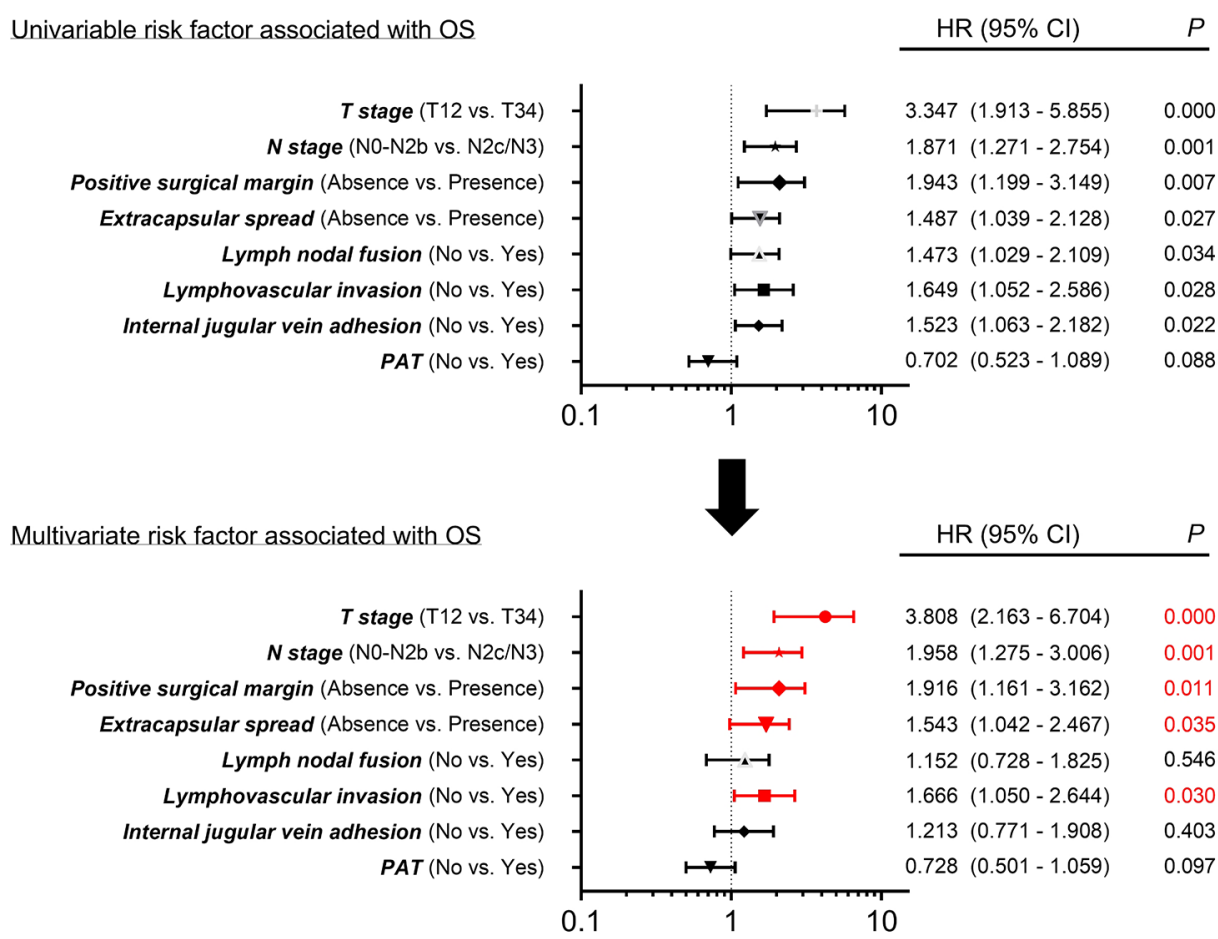

Figure 2 Univariate and multivariate analysis of factors associated with OS. OS, overall survival.
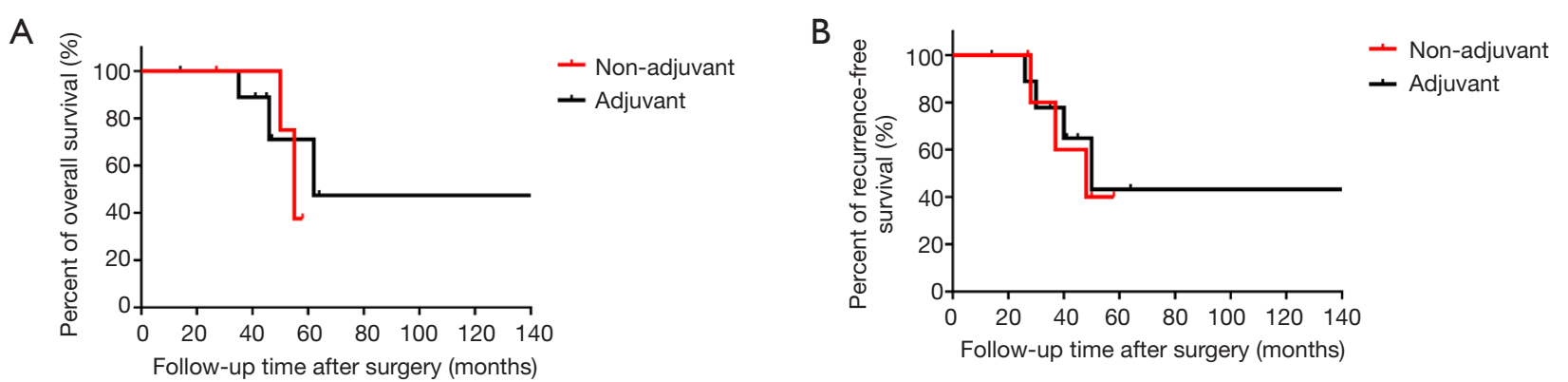

Figure 3 OS (A) and RFS (B) curves of stage IV patients without adverse features stratified by receiving postoperative adjuvant treatment (pRT or pCRT) or not. OS, overall survival; RFS, recurrence-free survival; pRT, plus postoperative radiotherapy; pCRT, plus postoperative chemoradiotherapy.

to pharyngolaryngectomy. However, some studies have reported that the long-term survival achieved by surgical treatment was superior to that achieved by non-surgical ones (15,17-20). Consideration of an optimal initial treatment for stage IV HSCC has not been assessed in any large, prospective and randomised study, thus, additional trials are needed to confirm the efficiency of a nonsurgical strategy for treating advanced HSCC. Here in our country, surgery-based strategies remain the most common therapeutic treatment for patients with stage IV HSCC.
Adjuvant RT is recommended for stage IV HSCC patients with advanced features, including positive surgical margins or extracapsular spread. Inadequate resection is reported to be correlated with increased risk of tumour recurrence and poorer survival outcome for patients (21-23). Recently, Harris has shown that all patients with involved margins had tumour recurrences no longer than 5 years after initial treatment, and the 5-year DFS of patients with negative margins was $48 \%$, which was significantly better than the DFS of patients with positive margins (24). In 


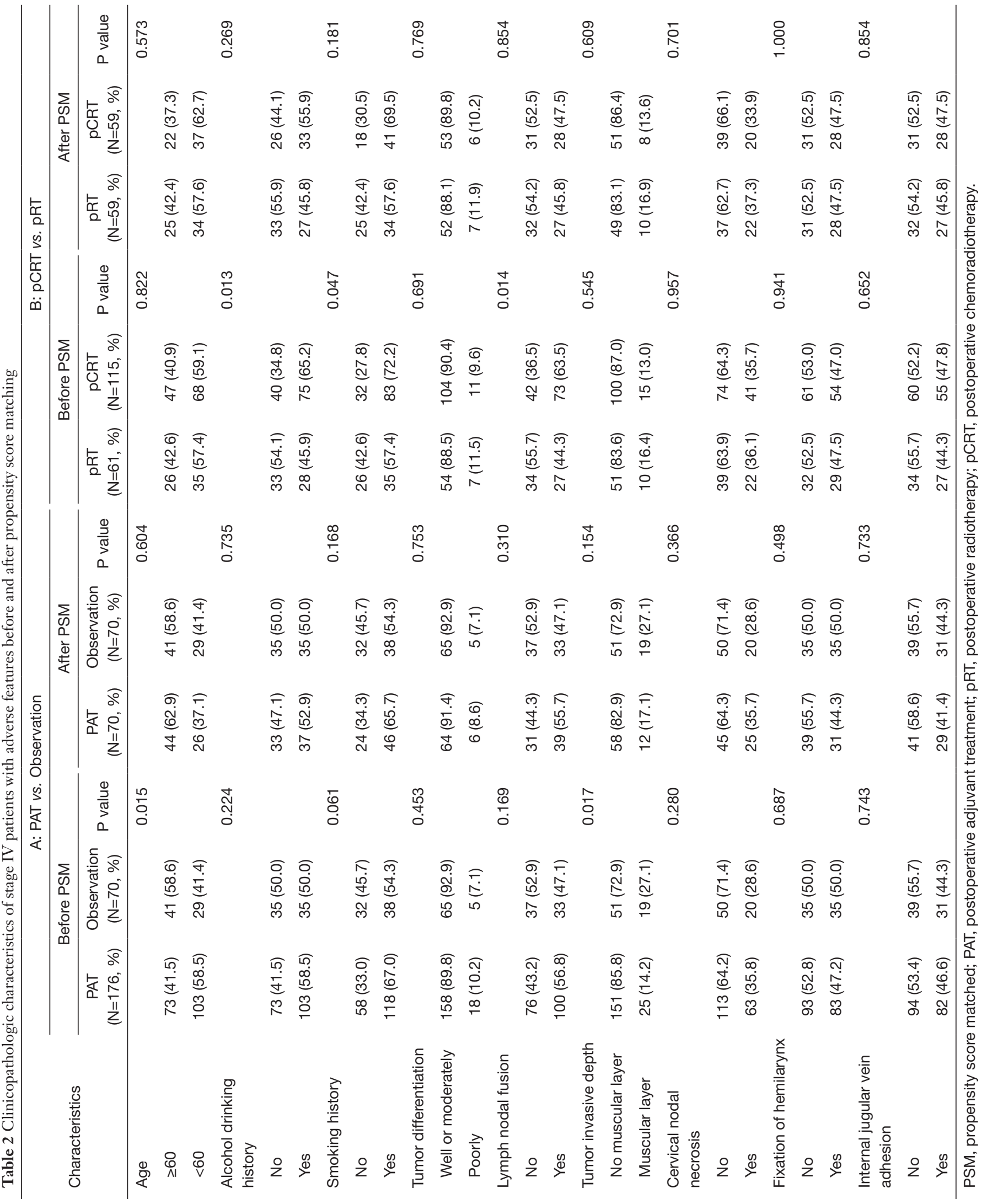


A Multivariate analysis of factors associated with os for matched patients (PAT vs. Observation)

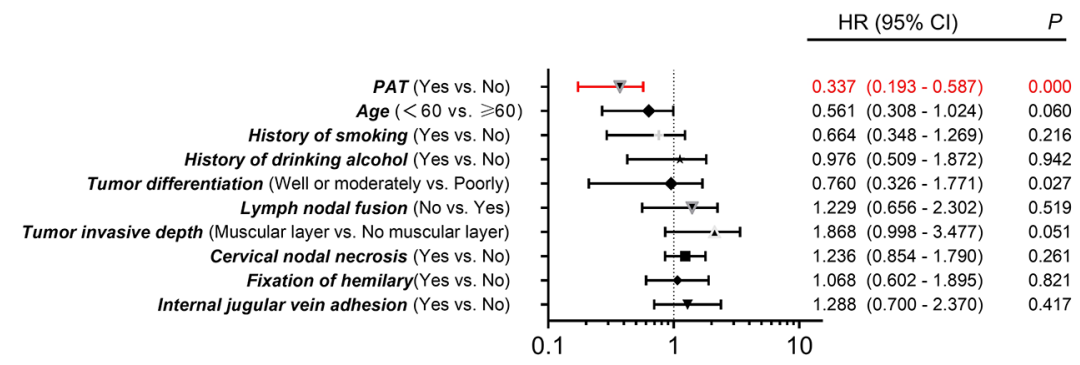

C

Multivariate analysis of factors associated with RFS for matched patients (PAT vs. Observation)

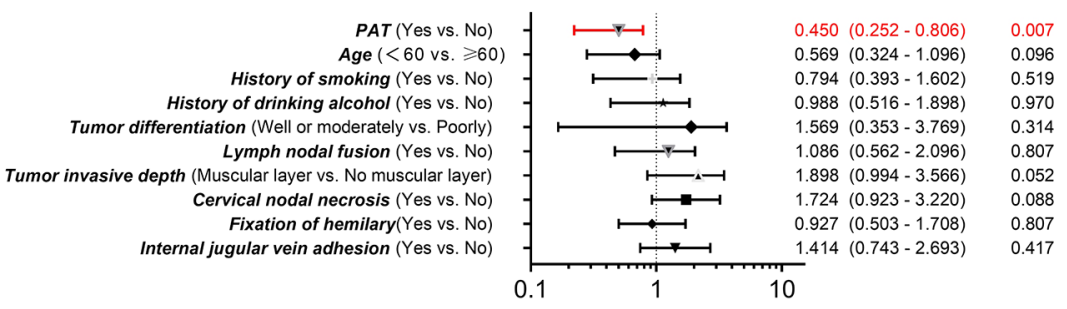

B

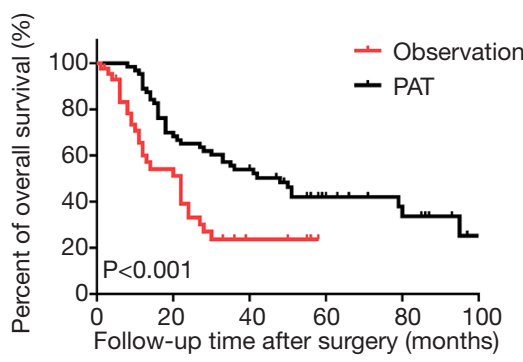

D

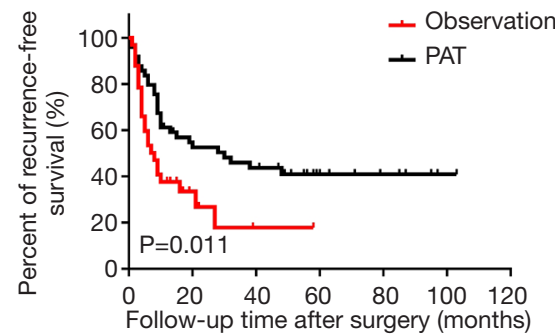

Figure 4 Multivariate analysis of factors associated with OS (A) and RFS (C) for matched patients from PAT group and Observation group; OS (B) and RFS (D) curves of stage IV patients with high-risk factors after PSM (70 pairs, PAT group vs. Observation group). OS, overall survival; RFS, recurrence-free survival; PSM, propensity score matching.

A Multivariate analysis of factors associated with os for matched patients ( $p R T$ vs. pCRT)

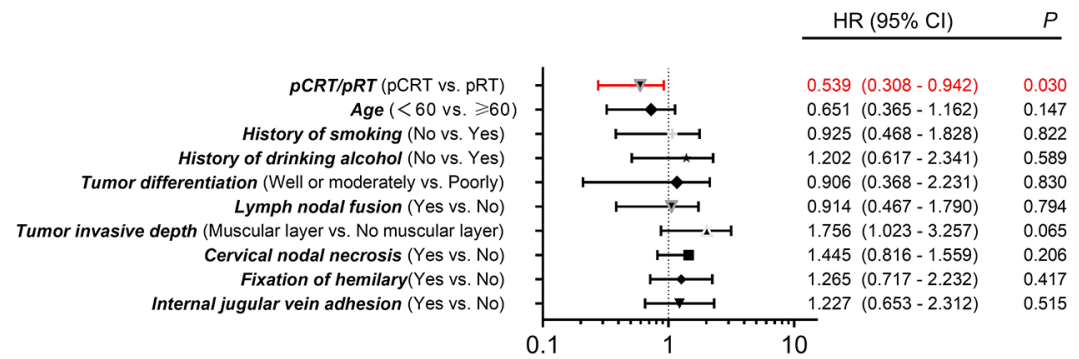

C Multivariate analysis of factors associated with RFS for matched patients (pRT vs. pCRT)

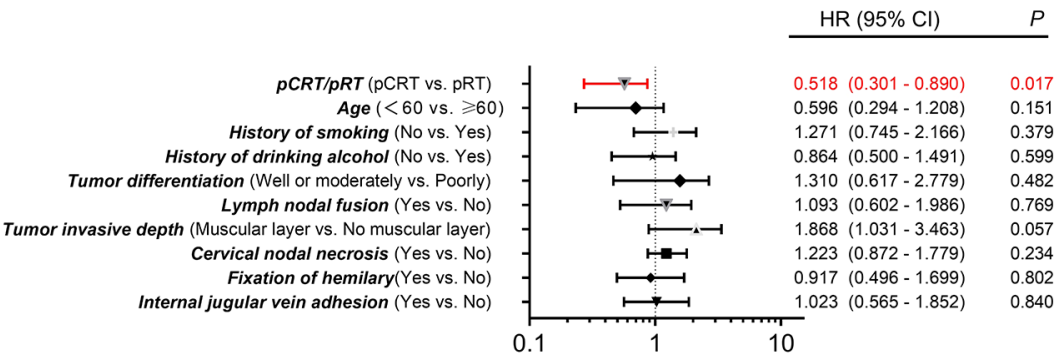

B

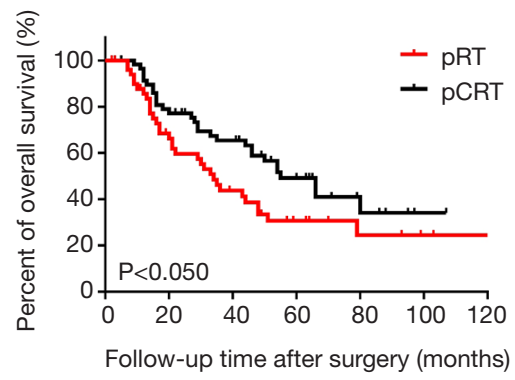

D

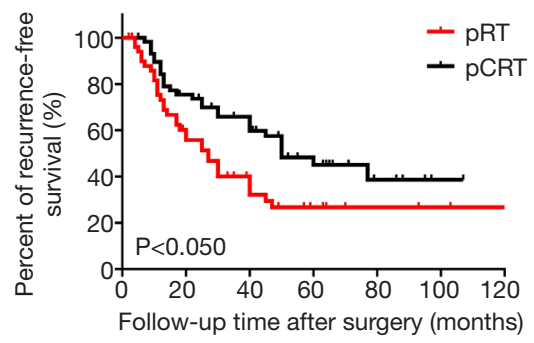

Figure 5 Multivariate analysis of factors associated with OS (A) and RFS (C) for matched patients from pRT group and pCRT group; OS (B) and RFS (D) curves of stage IV patients with high-risk factors after PSM (59 pairs, pRT group vs. pCRT group). OS, overall survival; RFS, recurrence-free survival; pRT, plus postoperative radiotherapy; pCRT plus postoperative chemoradiotherapy. 
our study, similar results were obtained; positive surgical margins were also significantly associated with worse OS rates on multivariate analysis. Submucosal extension has a high probability of presenting in HSCC. Recently, Jang et al. (25) found that there was a significant correlation between the degree of microscopic tumour extension from the gross tumour border and the sizes of primary tumour, with a median (range) of $0.84(0.14-2.32) \mathrm{mm}, 1.06(0.20$ 4.34) $\mathrm{mm}$ and $1.77(0.13-4.70) \mathrm{mm}$ in $\mathrm{T} 1, \mathrm{~T} 2$ and $\mathrm{T} 3-4$ stages respectively. Incidentally, most patients with stage IV HSCC are T3-4 stages (75.7\% in our cohort). Considering the high risk of recurrence, for patients with T4 tumours, large surgical ranges are commonly performed for adequate safe margins. But for T3 diseases, the surgical excision is relatively conservative for function retention, which may increase the possibility of positive margins. Given the worse survival outcome due to increased risk of positive margins in patients with stage IV HSCC, suitable postoperative management is crucial.

Previous studies have identified extracapsular spread, defined as a tumour extending outside the lymph node capsule, to be another negative prognostic factor for survival in patients with laryngeal and hypopharyngeal cancer $(26,27)$. In our study, the multivariate analysis results showed that the OS rate was significantly higher in patients without extracapsular spread. However, Coatesworth and MacLennan (28) reported that $10.5-25 \%$ of patients with $\mathrm{cN} 0$ disease were found to have extracapsular spread. Considering the poor survival outcome caused by extracapsular spread, the preoperative diagnosis of this factor seems crucial especially for those with clinically negative neck diseases. Nowadays, although diagnostic accuracy remains unsatisfactory, the combination of contrast-enhanced CT-scan and presence of central node necrosis is considered the most efficient diagnostic modality for clinical diagnosis of extracapsular spread (29). In our view, more specific indicators such as characteristic protein biomarkers for preoperative detection of extracapsular spread should be explored to achieve higher diagnostic accuracy. An internationally accepted grading criteria should also be created for differentiating the severity of extracapsular spread to help determine optimal treatment strategies for HSCC patients.

According to the 7 th American Joint Committee on Cancer (AJCC) Staging Manual (14), stage pT4 is considered to be a risk factor. Invasion of cricoid cartilage or central compartment soft tissue is classified as stage $\mathrm{T} 4 \mathrm{a}$, and invasion of the prevertebral fascia or mediastinal structures or encasing carotid artery is classified as T4b. In our study, among patients with stage IV HSCC, the survival outcomes of patients with stage T3 disease were found to be similar to those with stage T4 disease. Moreover, multivariate analysis confirmed that patients with stages T3-T4 showed a significant correlation with decreased OS. Stage T3 of this disease is defined as a tumour with a diameter $>4 \mathrm{~cm}$ or with fixation of hemilarynx or extension of the oesophagus. With stages T3-T4, a tumour usually means deeper infiltration and a large oesophagus extension, which could explain the results of our study that showed patients with stages T3-T4 HSCC had poor survival outcomes in terms of OS.

The level of lymph node metastasis (LNM) has been reported to be an independent prognostic factor for survival in patients with advanced HNSCC (30), and cervical lymph node management is an important factor that affects the outcomes of HSCC treatment (31). A previous study reported that, in locally advanced HNSCC, patients with metastatic lymph nodes classified as N2/N3 have poorer tumour outcomes than patients classified as N0/N1 (32). However, no previous study has focused on the treatment outcomes of HSCC patients with bulky $\mathrm{N}$ disease.

In this study, we paid attention to the outcomes of management for patients with different levels of LNM. A survival rate comparison between patients with different $\mathrm{N}$ stages showed that the survival outcome was worse for patients classified as $\mathrm{N} 3$ than patients classified as $\mathrm{N} 0-$ $\mathrm{N} 2$, and patients with $\mathrm{N} 2$ disease showed similar survival outcomes as those with $\mathrm{N} 0-\mathrm{N} 1$ disease. To further study bulky $\mathrm{N}$ disease amongst stage IV HSCC patients, patients with $\mathrm{N} 2$ disease were subdivided into N2a, N2b and N2c groups, and the Kaplan-Meier analysis of OS in patients with $\mathrm{N} 2-\mathrm{N} 3$ disease showed that OS was significantly worse for $\mathrm{N} 2 \mathrm{c} / \mathrm{N} 3$ patients than $\mathrm{N} 2 \mathrm{a} / \mathrm{N} 2 \mathrm{~b}$ patients. The multivariate analysis results also confirmed that patients with $\mathrm{N} 2 \mathrm{c} / \mathrm{N} 3$ disease had a significantly poorer OS than patients with N0-N2b disease. Patients with N2c disease all have bilateral or contralateral lymph nodal metastasis, while the metastatic lymph nodes are confined to one side in patients with $\mathrm{N} 2 \mathrm{a}$ disease and $\mathrm{N} 2 \mathrm{~b}$ disease, which may be the reason for the poor OS finding.

Lymphovascular invasion includes lymphatic invasion and vascular invasion. Michikawa et al. (33) reported that lymphovascular invasion can be used to predict lymph node metastasis, and is associated with poor prognosis. Similar results were obtained in our research. The discovery of lymphovascular invasion signifies a number of cancer cells 
were invading into the vascular cavity and may indicate the development of metastasis, which can explain the poorer survival outcome in patients with this factor. Saito (34) evaluated the correlation of lymphatic invasion and vascular invasion with treatment outcomes separately, and found vascular invasion was a strong prognostic biomarker for patients with advanced HSCC while lymphatic invasion was not. In view of the small sample size of their study, more multicenter, large-sample studies are needed to confirm the separate prognostic values of lymphatic and vascular invasion.

After determining the risk factors, treatment strategies should be discussed. According to the National Comprehensive Cancer Network guidelines, postoperative CRT is recommended after surgical resection for patients with high-risk adverse features, including positive surgical margins or extracapsular spread of nodal metastasis (35). However, for risk factors that are considered to be intermediate, such as pT3 or pT4 primary stages, N2 or $\mathrm{N} 3$ classification and lymphovascular invasion, there are no definite consensus on the use of postoperative chemotherapy, so explicit indications for postoperative treatment strategy remain undetermined. Several published studies have reported that PAT could provide satisfactory locoregional control and survival rates in locally advanced HNSCC $(36,37)$. Randomised trials have also indicated that postoperative CRT can result in a better survival outcome in HNSCC patients with adverse features, mainly including extracapsular spread of nodal disease and microscopically positive mucosal margins of resection $(38,39)$.

In this study, we determined the factors that significantly influence the survival outcomes of stage IV HSCC, including extracapsular spread and positive margins, which have been regarded as adverse features in most previous studies. We also concluded adverse features of pT3 and pT4 stages, N2c and N3 stages, and lymphovascular invasion that significantly impact survival outcomes. We found that PAT can significantly reduce the risk of recurrence and increase survival rate for patients in this group, but has little influence on survival outcomes in the non-adverse features group, as it is generally believed that RT can efficiently prevent cancer cell residual and microscopic deposits, which would lead to tumour recurrence if mismanaged (36). Recently, researchers have been investigating advanced techniques of RT, such as intensity modulated radiation therapy (IMRT) as an adjuvant strategy for HNSCC (40). However, information on IMRT in a postoperative setting for advanced HSCC is scarce; thus, additional studies are needed to evaluate the efficiency of this strategy.

In regards to use of chemotherapy, for patients with adverse features in our study, those who received pCRT (including CCRT and seq-CRT) achieved better RFS and better OR than patients who only received postoperative RT. Locoregional failure was the major recurrence pattern for advanced HNSCC patients according to previous report (41). Similarly, in our study, the majority of the recurrence patterns were locoregional failures (53.9\%) in patients with stage IV HSCC, indicating the high risk of tumour recurrence and metastasis in patients with this disease. Chemotherapy can efficiently kill residual cancer cells in subclinical metastatic lesions, which may explain the preferable survival outcome when using pCRT instead of only using pRT.

\section{Study limitations}

Our study has some limitations. First, selection bias is unavoidable due to the study's retrospective design and single institution participation. Although PSM was conducted, which can reduce bias to some extent in each group, some unknown confounders which were not included in the database may affect the outcomes, such as the patients' socioeconomic status. Second, in regards to lymphovascular invasion, we did not differentiate between vascular invasion and lymphatic invasion. It has been reported that vascular invasion is a stronger prognostic biomarker for advanced hypopharyngeal carcinoma (34); however, there are very few studies with a large sample size that aim to assess the separated influence of vascular invasion and lymphatic invasion in patients receiving tumour resection for hypopharyngeal carcinoma. Third, we administered 1-2 cycles of treatment for patients as an efficient chemotherapy strategy, but we did not investigate how the regimens and dosage affected the survival outcome. Fourth, details on the extent that smokers and drinkers engage in their respective activities are not known. We believe future multicentric and prospective studies are needed to validate our conclusions.

\section{Conclusions}

The listed factors are seen as adverse features: pT3 and pT4 stages, N2c and N3 classifications, positive surgical margins, extracapsular spread of nodal disease and lymphovascular invasion. For patients that possess these factors, PATs are necessary even though patients underwent 
complete resection with no evidence of residual cancer inspected macroscopically or microscopically. Of the possible PATs, pCRT is considered to be the best choice. However, for patients without these adverse features (T1$2 \mathrm{~N} 2 \mathrm{a}-2 \mathrm{~b}$, without positive surgical margins, extracapsular spread of nodal disease and lymphovascular invasion) PATs seem unnecessary; instead, observation and regular reexamination should suffice after tumour resection.

\section{Acknowledgments}

Funding: This study was supported by the Science and Technology Innovation Project of Shanghai Shenkang Hospital Clinical Development Center under Grand [SHDC12015114]; the Science and Technology Commission of Shanghai Municipality under Grand [16411950100]; the National Natural Science Foundation of China under Grand [81772878, 30801283, 30972691]; the Shanghai Science and Technology Development Funds under Grand [09QA1401000, 10QA1405900, 14411961900]; the Training Program of the Excellent Young Talents of Shanghai Municipal Health System under Grand [XYQ2011055, XYQ2011015]; and the Shanghai Municipal Science and Technology Foundation under Grand [11JC1410802].

\section{Footnote}

Conflicts of Interest: The authors have no conflicts of interest to declare.

Ethical Statement: The authors are accountable for all aspects of the work in ensuring that questions related to the accuracy or integrity of any part of the work are appropriately investigated and resolved. This study was approved by the Institutional Ethics Committee of the Eye \& ENT Hospital of Fudan University (No. KJ2008-01). All participants gave informed consent to take part in the study.

Open Access Statement: This is an Open Access article distributed in accordance with the Creative Commons Attribution-NonCommercial-NoDerivs 4.0 International License (CC BY-NC-ND 4.0), which permits the noncommercial replication and distribution of the article with the strict proviso that no changes or edits are made and the original work is properly cited (including links to both the formal publication through the relevant DOI and the license). See: https://creativecommons.org/licenses/by-nc-nd/4.0/.

\section{References}

1. Hall SF, Groome PA, Irish J, et al. The natural history of patients with squamous cell carcinoma of the hypopharynx. Laryngoscope 2008;118:1362-71.

2. Wahlberg PC, Andersson KE, Biorklund AT, et al. Carcinoma of the hypopharynx: analysis of incidence and survival in Sweden over a 30-year period. Head Neck 1998;20:714-9.

3. Buckley JG, Maclennan K. Cervical node metastases in laryngeal and hypopharyngeal cancer: a prospective analysis of prevalence and distribution. Head Neck 2000;22:380-5.

4. Keereweer S, Kerrebijn JD, Al-nangani A, et al. Chemoradiation for advanced hypopharyngeal carcinoma: a retrospective study on efficacy, morbidity and quality of life. Eur Arch Otorhinolaryngol 2012;269:939-46.

5. Takes RP, Strojan P, Silver CE, et al. Current trends in initial management of hypopharyngeal cancer: the declining use of open surgery. Head Neck 2012;34:270-81.

6. Tulunay-Ugur OE, Mcclinton C, Young Z, et al. Functional outcomes of chemoradiation in patients with head and neck cancer. Otolaryngol Head Neck Surg 2013;148:64-8.

7. Vokes EE, Kies MS, Haraf DJ, et al. Concomitant chemoradiotherapy as primary therapy for locoregionally advanced head and neck cancer. J Clin Oncol 2000;18:1652-61.

8. Brockstein B, Haraf DJ, Rademaker AW, et al. Patterns of failure, prognostic factors and survival in locoregionally advanced head and neck cancer treated with concomitant chemoradiotherapy: a 9-year, 337-patient, multiinstitutional experience. Ann Oncol 2004;15:1179-86.

9. Reis I, Aguiar A, Alzamora C, et al. Locally advanced hypopharyngeal squamous cell carcinoma: singleinstitution outcomes in a cohort of patients curatively treated either with or without larynx preservation. Radiol Bras 2016;49:21-5.

10. Qian W, Zhu G, Wang Y, et al. Multi-modality management for loco-regionally advanced laryngeal and hypopharyngeal cancer: balancing the benefit of efficacy and functional preservation. Med Oncol 2014;31:178.

11. Kuo P, Chen MM, Decker RH, et al. Hypopharyngeal cancer incidence, treatment, and survival: temporal trends in the United States. Laryngoscope 2014;124:2064-9.

12. Chen LY, Huang CC, Tsou YA, et al. Prognostic factor of severe complications in patients with hypopharyngeal cancer with primary concurrent chemoradiotherapy. 
Anticancer Res 2015;35:1735-41.

13. Habib A. Management of advanced hypopharyngeal carcinoma: systematic review of survival following surgical and non-surgical treatments. J Laryngol Otol 2018;132:385-400.

14. National Comprehensive Cancer Network. (NCCN) Clinical Practice Guidelines in Oncology. Head and Neck Cancer, Version 2. 2017. Available online: https://www. nccn.org/professionals/physician_gls/

15. Newman JR, Connolly TM, Illing EA, et al. Survival trends in hypopharyngeal cancer: a population-based review. Laryngoscope 2015;125:624-9.

16. Edge SB, Compton CC. The American Joint Committee on Cancer: the 7 th edition of the AJCC cancer staging manual and the future of TNM. Ann Surg Oncol 2010;17:1471-4.

17. Tsou YA, Lin MH, Hua CH, et al. Survival outcome by early chemoradiation therapy salvage or early surgical salvage for the treatment of hypopharyngeal cancer. Otolaryngol Head Neck Surg 2007;137:711-6.

18. Krstevska V, Stojkovski I, Lukarski D. Concurrent radiochemotherapy in advanced hypopharyngeal cancer. Radiat Oncol 2010;5:39.

19. Kuo YL, Chang CF, Chang SY, et al. Partial laryngopharyngectomy in the treatment of squamous cell carcinoma of hypopharynx: analysis of the oncologic results and laryngeal preservation rate. Acta Otolaryngol 2012;132:1342-6.

20. Harris BN, Biron V L, Donald P, et al. Primary Surgery vs Chemoradiation Treatment of Advanced-Stage Hypopharyngeal Squamous Cell Carcinoma. JAMA Otolaryngol Head Neck Surg 2015;141:636-40.

21. Beitler JJ, Smith RV, Silver CE, et al. Close or positive margins after surgical resection for the head and neck cancer patient: the addition of brachytherapy improves local control. Int J Radiat Oncol Biol Phys 1998;40:313-7.

22. Ravasz LA, Slootweg PJ, Hordijk GJ, et al. The status of the resection margin as a prognostic factor in the treatment of head and neck carcinoma. J Craniomaxillofac Surg 1991;19:314-8.

23. Spiro RH, Guillamindegui OJ, Paulino AF, et al. Pattern of invasion and margin assessment in patients with oral tongue cancer. Head Neck 1999;21:408-13.

24. Kim SY, Rho YS, Choi EC, et al. Clinicopathological factors influencing the outcomes of surgical treatment in patients with T4a hypopharyngeal cancer. BMC Cancer 2017;17:904.

25. Jang JY, Choi N, Ko YH, et al. Differential Impact of
Close Surgical Margin on Local Recurrence According to Primary Tumor Size in Oral Squamous Cell Carcinoma. Ann Surg Oncol 2017;24:1698-706.

26. Prim MP, De Diego JI, Hardisson D, et al. Extracapsular spread and desmoplastic pattern in neck lymph nodes: two prognostic factors of laryngeal cancer. Ann Otol Rhinol Laryngol 1999;108:672-6.

27. Brasilino de Carvalho M. Quantitative analysis of the extent of extracapsular invasion and its prognostic significance: a prospective study of 170 cases of carcinoma of the larynx and hypopharynx. Head Neck 1998;20:16-21.

28. Coatesworth AP, MacLennan K. Squamous cell carcinoma of the upper aerodigestive tract: the prevalence of microscopic extracapsular spread and soft tissue deposits in the clinically N0 neck. Head Neck 2002;24:258-61.

29. Mermod M, Tolstonog G, Simon C, et al. Extracapsular spread in head and neck squamous cell carcinoma: A systematic review and meta-analysis. Oral Oncol 2016;62:60-71.

30. Howell GM, Grandis JR. Molecular mediators of metastasis in head and neck squamous cell carcinoma. Head Neck 2005;27:710-7.

31. Layland MK, Sessions DG, Lenox J. The influence of lymph node metastasis in the treatment of squamous cell carcinoma of the oral cavity, oropharynx, larynx, and hypopharynx: N0 versus $\mathrm{N}+$. Laryngoscope 2005;115:629-39.

32. Xing Y, Zhang J, Lin H, et al. Relation between the level of lymph node metastasis and survival in locally advanced head and neck squamous cell carcinoma. Cancer 2016;122:534-45.

33. Michikawa C, Uzawa N, Kayamori K, et al. Clinical significance of lymphatic and blood vessel invasion in oral tongue squamous cell carcinomas. Oral Oncol 2012;48:320-4.

34. Saito Y, Omura G, Yasuhara K, et al. Prognostic value of lymphovascular invasion of the primary tumor in hypopharyngeal carcinoma after total laryngopharyngectomy. Head Neck 2017;39:1535-43.

35. Colevas AD, Yom SS, Pfister DG, et al. NCCN Guidelines Insights: Head and Neck Cancers, Version 1.2018. J Natl Compr Canc Netw 2018;16:479-90.

36. Langendijk JA, Ferlito A, Takes RP, et al. Postoperative strategies after primary surgery for squamous cell carcinoma of the head and neck. Oral Oncol 2010;46:577-85.

37. Hinerman RW, Morris CG, Amdur RJ, et al. Surgery and postoperative radiotherapy for squamous cell carcinoma of 
the larynx and pharynx. Am J Clin Oncol 2006;29:613-21.

38. Cooper JS, Pajak TF, Forastiere AA, et al. Postoperative concurrent radiotherapy and chemotherapy for high-risk squamous-cell carcinoma of the head and neck. N Engl J Med 2004;350:1937-44.

39. Bernier J, Domenge C, Ozsahin M, et al. Postoperative irradiation with or without concomitant chemotherapy for locally advanced head and neck cancer. N Engl J Med
2004;350:1945-52.

40. Duprez F, Bonte K, De Neve W, et al. Regional relapse after intensity-modulated radiotherapy for head-and-neck cancer. Int J Radiat Oncol Biol Phys 2011;79:450-8.

41. Goto Y, Kodaira T, Furutani K, et al. Clinical outcome and patterns of recurrence of head and neck squamous cell carcinoma with a limited field of postoperative radiotherapy. Jpn J Clin Oncol 2013;43:719-25.
Cite this article as: Heng Y, Zhu X, Zhou L, Zhang M, Zhou $\mathrm{H}$, Tao L. The presence of risk factors and corresponding treatment strategies post-surgical resection in stage IV hypopharyngeal squamous cell carcinoma patients: a retrospective cohort study. Ann Transl Med 2020;8(5):189. doi: 10.21037/atm.2020.01.102 


\section{Supplementary}

Table S1 Treatment characteristics of all patients

\begin{tabular}{|c|c|c|}
\hline Treatment & $\mathrm{n}$ & $\%$ \\
\hline LF preservation laryngectomy & 61 & 22.8 \\
\hline Total laryngectomy & 206 & 77.2 \\
\hline \multicolumn{3}{|l|}{ Type of neck dissection } \\
\hline Bilateral & 51 & 19.1 \\
\hline \multicolumn{3}{|l|}{ Postoperative adjuvant therapy } \\
\hline pCRT (CCRT/seq-CRT/unknow) & $69 / 42 / 12$ & 46.1 \\
\hline RT alone & 48 & 18.0 \\
\hline
\end{tabular}

pCRT, postoperative chemoradiotherapy; CCRT, concurrent chemoradiotherapy; CRT, chemoradiotherapy.

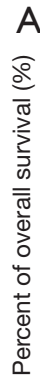

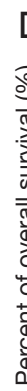

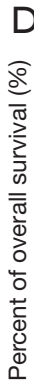

G

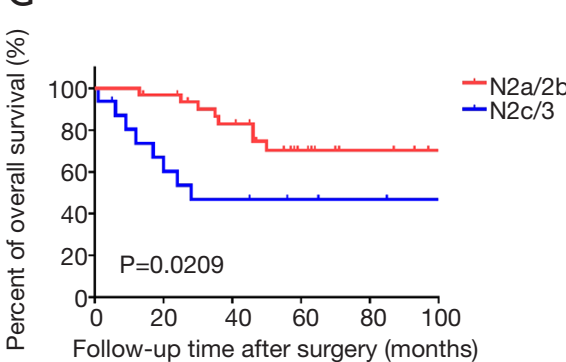

(20)

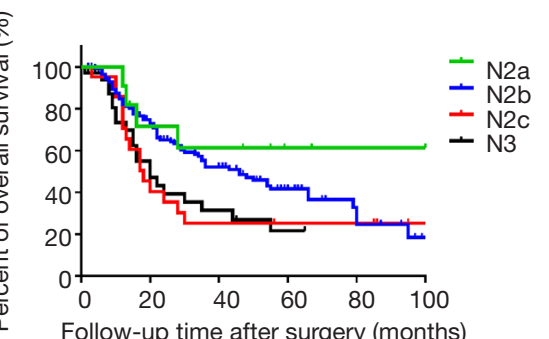

Figure S1 OS curves of stage IV HSCC patients. (A,B) OS curves of patients with stage IV HSCC stratified by T-classification; (C,D,E) OS curves of patients with stage IV HSCC stratified by N-classification; (F,G) OS curves of stage IVHSCC patients with T1/2 stage stratified by N-classification: (H,I); OS curves of stage IV HSCC patients with T3/4 stage stratified by N-classification.
B

웅
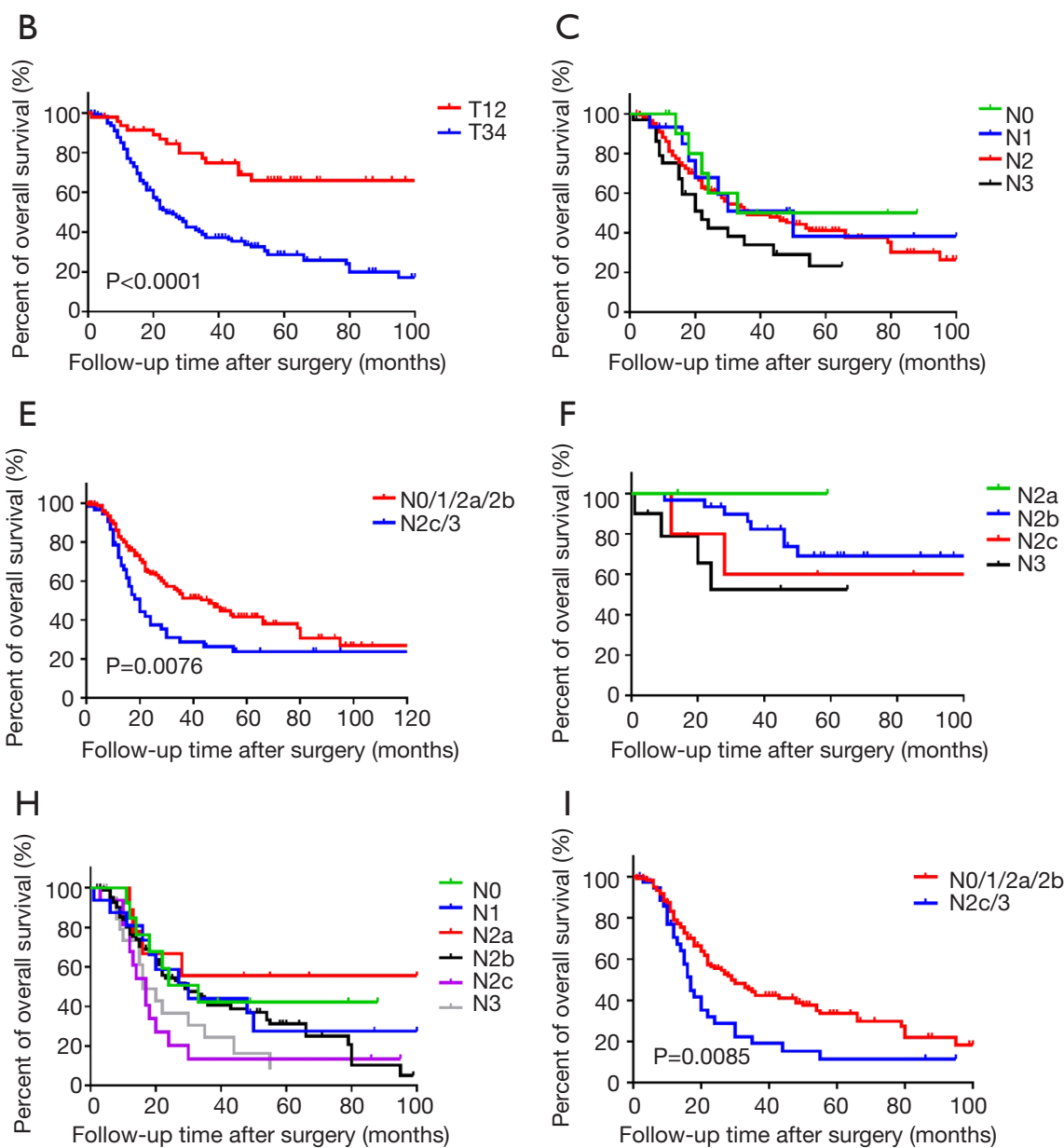

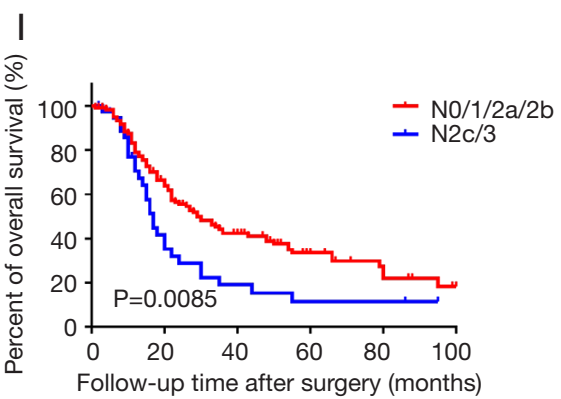


Table S2 Patterns of recurrence

\begin{tabular}{lcc}
\hline Recurrent site & No. & $\%$ \\
\hline All & 154 & 100.0 \\
Primary & 19 & 12.3 \\
Locoregional & 83 & 53.9 \\
Distant & 52 & 33.8 \\
Lung & 33 & 21.4 \\
Liver & 8 & 5.2 \\
Bone & 11 & 7.1 \\
Brain & 4 & 2.6 \\
Intestine & 1 & 0.6 \\
Esophagus & 5 & 3.2 \\
Other & 3 & 1.9 \\
\hline
\end{tabular}

